\title{
Focus groups show need for diabetes awareness education among African Americans
}

\section{UC Health Promotion Workgroup}

\section{The UC Division of Agriculture and} Natural Resources Health

Promotion Workgroup assessed diabetes awareness among African Americans at risk for the disease. Workgroup members conducted focus group discussions with the target population in Los Angeles, San Bernardino, San Joaquin, San Mateo, Santa Clara and Stanislaus counties. Although obesity is considered a major risk factor for type 2 diabetes, most participants cited poor dietary patterns, rather than body weight, as the most important factor in the high rate of diabetes among African Americans. Food preferences, family pressure and lack of social support were most often mentioned as obstacles to healthful dietary changes. Many felt that not enough information about diabetes was reaching the black community and voiced the need for culturally sensitive education, delivered through community-based channels.

$\mathrm{O}$ ver the past two decades, the prevalence of diabetes in the United States has increased by $33 \%$, from $4.9 \%$ in 1990 to $6.5 \%$ in 1998 (Mokdad et al. 2000). Concurrently, California has experienced an increase of $67 \%$ in the prevalence of diabetes, from $4.1 \%$ to $6.9 \%$. Experts believe these trends are due to the "obesity epidemic," as excess body weight is a major risk factor for type 2 diabetes.

Generally developing later in life, type 2 diabetes accounts for $90 \%$ to $95 \%$ of all cases and is characterized by insulin resistance and/or inability of the pancreas to deliver insulin in a regulated way to the cells. Type 1 diabetes, usually diagnosed during child-

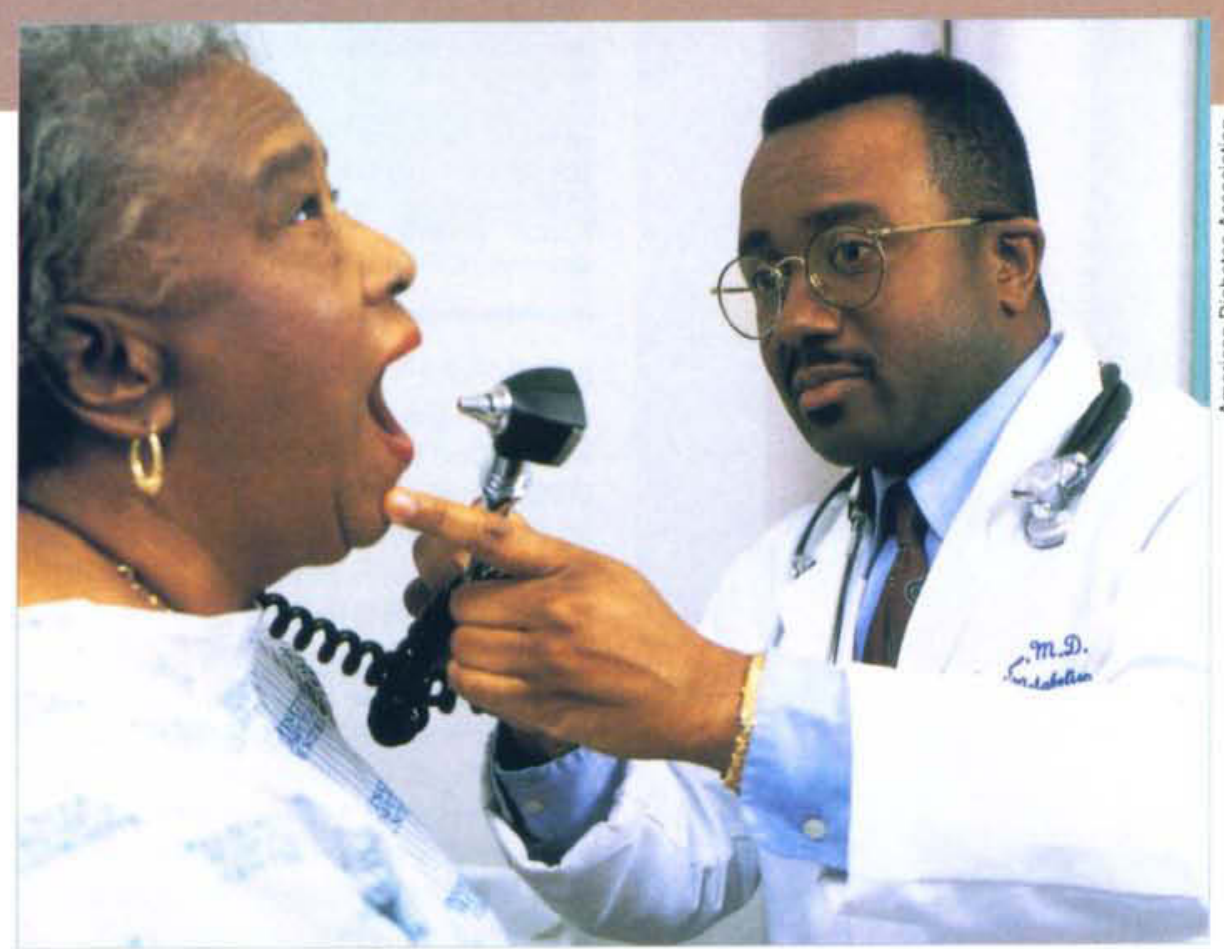

The rate of type 2 diabetes is $\mathbf{1 . 6}$ times higher among blacks than whites; about $\mathbf{2 5 \%}$ of African American women over age $\mathbf{5 5}$ suffer from the disease.

hood or adolescence, involves an autoimmune process that destroys the insulin-producing cells in the pancreas. Those with type 1 diabetes must inject insulin, while people with type 2 usually control their diabetes through proper diet, exercise and/or oral medication. However, as the disease progresses or during pregnancy, even those with type 2 diabetes may need insulin.

The American Diabetes Association estimates that the direct and indirect costs related to all types of diabetes were more than $\$ 98$ billion in 1997. Early detection and careful management of all types of diabetes through diet, physical activity and medication can lower the risk of complications and improve the quality of life.

The prevalence of type 2 diabetes is significantly higher in Native Americans, Latinos and African Americans than in non-Latino whites. Native Americans and Mexican Americans are two to five times more likely to have diabetes than non-Latino whites
(Hosey et al. 1998, Harris et al. 1998). Although these two ethnic groups have a disproportionate rate of diabetes, the rate is 1.6 times higher in blacks than whites. In women, the rates are especially high: About one in four African American women over 55 has type 2 diabetes.

A number of factors may contribute to the increased risk of diabetes and diabetes-related complications in African Americans. In a study of 975 whites and 418 blacks, obesity accounted for $47 \%$ of the excess risk of diabetes in black women (Brancati et al. 2000). Genetic factors are also likely to play a role, but more research related to genetic and environmental influences is needed (Pi-Sunyer 1990,

For more information about diabetes, consult with your doctor or contact the American Diabetes Association, at 1-800-DIABETES (1-800-342-2383), or visit the Association's Web site at www.diabetes.org. 
TABLE 1: Participant responses, diabetes focus groups $(n=60)$

Perceived cause:
Poor diet
Genetics/family trait
Stress
Lack of exercise
Lack of awareness
Lack of medical attention
Don't know
Other* (obesity, high cholesterol)
Perceived barriers to change:
Food preferences
Family pressure
Lack of social support
Forgetfulness
Lack of education
Age
Other (too much food in this
society, overwhelmed, not enough
money to go to a gym, lack of stores)

Perceived obstacles to health care: Lack of cultural sensitivity/respect

Time/convenience/hours

Other (lack of insurance, fear. lack of free screenings)

Preferred educational channels: Community-based outreach programs (community centers, gym, YMCA health fairs, cooking classes)

Health providers

Schools

Churches

Peer counselors

Internet/Web

Brochures

Direct mail

Other (e-mail, videos, hotlines, restaurant 1 billboards, buses, family, commercials, newspaper)

- All factors listed in "other" categories were mentioned one time in the discussion.

Schneider et al. 1997, Osei et al. 1993). Poor nutrition during pregnancy, resulting in newborns with low birth weights, may also contribute to increased risk of diabetes later in life. Low socioeconomic status may limit access to medical care and healthful foods, and increase sedentary behavior, but higher educational and income levels do not always have a protective effect in African Americans (Gaillard et al. 1997). Racial discrimination, which has been linked to hypertension in African Americans, may undermine health promotion efforts and could also be a factor (Krieger and Sidney 1996).

A recent study involving a multicenter, randomized, controlled trial reported that intensive lifestyle changes can reduce the incidence of type 2 dia-
Lifestyle modifications, such as a healthier diet, weight management and moderate physical activity, can delay the onset of diabetes. Using focus groups, UC researchers examined the perceived causes of diabetes and barriers to change among African Americans who had not been diagnosed with the disease.

betes over a 3-year period by as much as $58 \%$ in populations at risk (Diabetes Prevention Program Research Group 2002). These lifestyle changes included 30 minutes of moderate physical activity on most days of the week, a weight loss of $7 \%$ and a low-fat diet.

Sustaining intensive lifestyle changes over the long term may be difficult without agricultural and environmental developments that increase public access to a low-fat diet and encourage physical activity. Nevertheless, if lifestyle changes could delay the onset of diabetes by only a few years, the personal benefits and savings in health care costs would be substantial. For example, during the first year after diagnosis, health care costs per person are $100 \%$ higher for people with diabetes compared with those who don't have diabetes. Eight years after diagnosis, costs are $133 \%$ higher (Brown et al. 1999). However, to reduce diabetes rates in minority populations at risk, more research is needed to develop culturally sensitive approaches to communication and education (Diabetes Research Working Group 1999).

\section{Assessing needs and perceptions}

A workgroup of UC faculty, Cooperative Extension specialists and county advisors conducted a series of focus groups with African American adults to examine beliefs and perceptions about diabetes. This work was carried out in preparation for a larger campaign to increase awareness and reduce risk factors in the same population. The focus groups took place in Los Angeles, San Bernardino, San Joaquin, San Mateo, Santa Clara and Stanislaus counties. Workgroup mem-

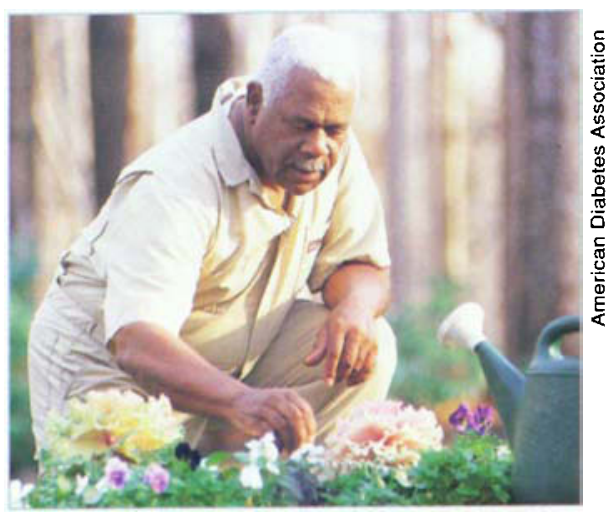

bers developed the research protocol, formulated focus group questions and interpreted the results. Through phone calls, church bulletins and personal contacts with agency staff and pastors, advisors recruited a convenience sample of participants from churches, clinics, housing projects and other community organizations. To be included in the study, participants had to be African American, between 20 and 50 years old and have not received a diagnosis of diabetes.

Seven focus groups (two in Los Angeles, one in each of the five other counties) were conducted among African American adults in church or clinic settings. The same person, an

\section{Focus group responses: Causes of diabetes}

"I don't think it is hereditary. It's eating habits handed down generation to generation."

- Santa Clara County

"We don't get the prevention and education ahead of time, so it passes down through generations. We have family that came here from Louisiana. They brought that part with them - the history, the eating and the cooking. It starts when we are infants: 'Give that baby some chili beans and corn bread. Feed that baby.'" - San Joaquin County

"A lot of different problems, especially prevalent among African Americans, could be attributed to food and stress. You eat when you are stressed or tired."

$$
\text { - Santa Clara County }
$$




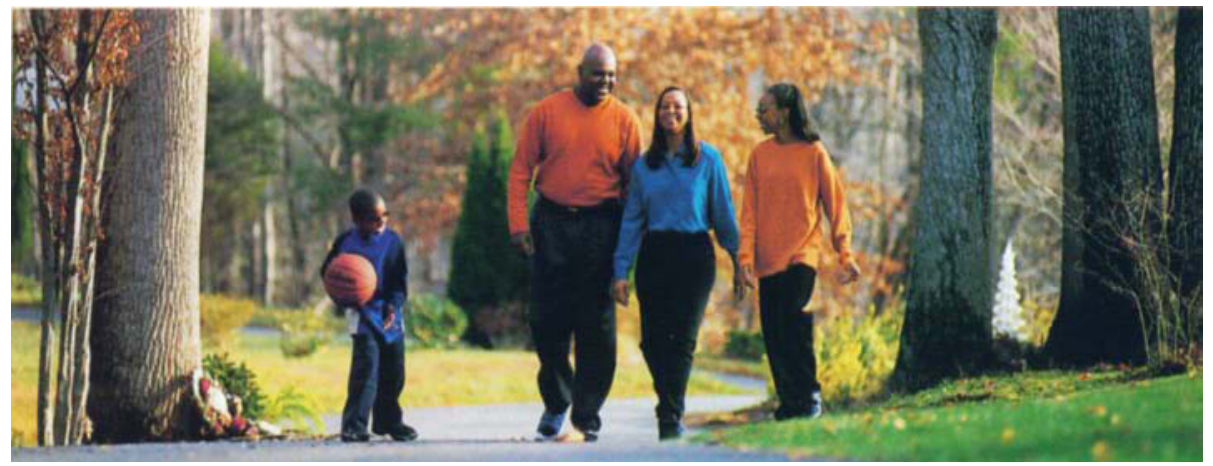

Family pressure and social support in the workplace were often cited as barriers to changing eating habits or losing weight.

African American woman, moderated each group to maintain consistency and establish a comfortable and friendly environment that encouraged the open sharing of ideas and opinions. In all but one county, an advisor acted as a co-moderator. The moderator followed a standardized focus group protocol, using open-ended questions, probes and cues, as recommended by R.A. Krueger (1994). The participants were offered refreshments and given a $\$ 25$ incentive to attend the sessions.

All sessions, except those in Los Angeles, were tape-recorded by the co-moderators and later transcribed by a graduate student with the UC Davis nutrition department. In Los Angeles, only the notes were available for analysis. Each of the co-moderators was asked to cross-check the transcripts from his or her county and provide overall impressions of the emerging themes at a workgroup meeting. A nutrition specialist coded and categorized segments of the transcripts using the QSR Nud*ist Version 4 software (Sage Publications, Thousand Oaks, CA). The moderator and another nutrition specialist independently hand-coded and categorized segments to determine the major and minor themes.

The focus groups began with general introductory questions about what people already know about diabetes (what they may have heard from others; whom do they know with the disease). Transition questions focused on factors contributing to the high rate of diabetes among African Americans and the importance of screening. The key questions concerned what advice they would give others who have symptoms, any obstacles they face in changing health practices and awareness of community resources related to diabetes. Final questions concerned how people prefer to receive information and what specific diabetes-related topics should be addressed (table 1).

\section{Focus on dietary role}

Sixty African American adults participated in the study: $30 \%(n=18)$ in Los Angeles County, $18 \%(n=11)$ in San Bernardino, $12 \%(\mathrm{n}=7)$ in San Joaquin, $15 \%(n=9)$ in San Mateo, $7 \%$ $(n=4)$ in Santa Clara and $18 \%(n=11)$ in Stanislaus. The average age was 32 years, ranging from 20 to 50 . None of the participants had been diagnosed with diabetes, although at least one had had gestational diabetes. Forty percent $(n=24)$ had at least one relative or family member with diabetes. Most of the participants were native Californians $(62 \%, n=37)$. Eighty-two percent $(n=49)$ of the participants were female and $18 \%(n=11)$ male. According to the advisors who recruited for the study, the groups included all low- and middle-income persons.

Perceived causes of diabetes. Although many people were uncertain about the cause of diabetes, dietary factors were mentioned most often as contributing to a high rate among African Americans. Most pointed to general eating habits - too much sugar, fat and "soul food." Several talked about their love for the spicy or wellseasoned foods prepared by other family members. Some talked about the link between current eating patterns and traditional foods passed down through generations since slavery. About equal numbers mentioned lack of exercise and stress as contribut-
Focus group responses: Barriers to change

"My fiancé is from the South and loves fried chicken. I am trying to lose weight, and it is tough to feed him. It doesn't matter how much seasoning or whatever I put on it when my heart wants fried chicken or fish."

- Stanislaus County

"In family gatherings, people are not supposed to diet but are supposed to eat their foods. There is that kind of family pressure." - San Mateo County

"People bring in lots of sweets to work, and even if you don't want any, you might one day give in and eat one donut, which increases to two and so on. Also, at home, when the rest of the family is eating fried chicken and sweets it makes dieting difficult." - San Bernardino County

ing factors. One person commented that African Americans were not targeted for messages that encourage physical activity. Although almost half $(n=24)$ had at least one immediate family member with diabetes, some participants were uncertain about the link between heredity (genetics) and diabetes. Being overweight was mentioned only once as a contributing factor. Further research is needed to clarify how the target group links poor dietary patterns to diabetes.

Barriers to change. Frequently, participants mentioned the difficulty of changing eating habits such as by limiting the intake of fat and sugar and avoiding cultural foods. Several mentioned family pressure, particularly at celebrations, and a lack of social support in the workplace. Other obstacles mentioned were age, lack of stores offering a variety of healthy foods in minority neighborhoods and "too much food in this society." One mentioned not having enough money to go to a gym.

Obstacles to health care. Lack of cultural sensitivity and respect among health providers was mentioned most 
Focus group responses: Obstacles to health care

"Even at the doctor's, you see nothing [about diabetes]. You see AIDS, breast cancer, lung cancer. How are we going to fight back, because we don't have anything to fight back with? We don't know anything." - San Joaquin County

"Race is an issue. Minorities are not treated on even par. We need to be educated to become more responsible and take responsibility for our health."

- Los Angeles County

"Don't come out here with the diabetes information on wheels. It's a different person each week. If they put that on wheels, that is not enough consistency. That is lack of respect for a person." - San Joaquin County

often as a barrier to early screening and preventive health care. Awareness of county-based resources related to diabetes varied widely. In San Joaquin County, participants were particularly vocal about obstacles to care, and no one was able to cite any relevant community resources. Although most viewed early screening as very important and would encourage anyone having symptoms to see a doctor, participants also expressed distrust and dissatisfaction with the health care system. Many felt they were not receiving information about diabetes or even being screened for the condition. Other factors mentioned were lack of free screenings, fear, time, inconvenience and lack of awareness.

Preferred channels for education. Four channels mentioned most often were community-based organizations (including health fairs, gyms, cooking classes), health providers, schools and churches. Participants were divided on the usefulness of disseminating diabetes materials via schools to parents, but agreed that children should be receiving this information early in their education. Opinions were also divided about the benefits of direct mail. Other channels mentioned were the Web or e-mail, posters on buses, billboards, public service announcements, family members, peer counselors, hotlines, brochures and restaurants. The diversity of channels mentioned probably reflects the differences in socioeconomic status and age among the groups.

Participants emphasized the need for information to be presented well. Effective approaches included using trained African Americans from the communities to take the message to the people. One participant suggested using the term "if you have sugar" to help people link their symptoms to diabetes and the need for screening. Being able to talk to people who have diabetes was also viewed as important.

Desired information. Many people knew something about the symptoms and dire consequences of diabetes, but their comments also reflected a lot of misinformation, such as that eating too much sugar causes diabetes. Most people wanted more information on causes, prevention and symptoms. They also wanted to know who's at risk, the types of diabetes, how often they should be screened, if diabetes is curable and the progression of the disease. Other information sought included: how to deal with diabetic coma, effect of diabetes on bones, how to eat right, lifestyle tips, alternative foods, new research on diabetes, how to control diabetes, how to lose weight and community resources.

\section{Interventions and next steps}

Since few in this study specifically linked being overweight with diabetes, interventions to prevent diabetes in African Americans might be more culturally acceptable - and possibly more effective - if issues related to dietary practices, the flavor and appeal of foods, social support and physical activity were addressed first. Many participants in our study also felt they had not received enough information about diabetes from the usual channels, given the relatively high risk of diabetes in the African American community. Participants also emphasized the need for culturally sensitive, community-based approaches to health promotion.
Focus group responses: Receiving education

"Church is a good place. You are already there. Have a nurse sit at the table and give you information." - Santa Clara County

"I want it to be reinforced, so I think it should come from the doctor or someone I can trust, like my OB [obstetrician]." - Santa Clara County

"Community fairs and things like that. I will go and listen to what they say. I have this thing with paper lately - I don't like to take the stuff anymore, but I will listen to somebody." - -Stanislaus County

"You can send all the fliers you want into the black community, but if it's not presented right, you can hang it up, they will not listen." - San Joaquin County

Findings from focus groups always should be viewed as qualitative and not necessarily representative of the population at large. The participants in our focus group discussions may have been more interested in the topic than others who did not volunteer for the study. Issues raised in certain groups may also have depended on the level of comfort participants felt in that setting.

Nonetheless, studies conducted among African Americans in other states have described similar themes. Particularly among older adults, the history of slavery is perceived to influence food choices (Airhihenbuwa et al. 1996). A major issue in diabetes management is the importance of food and eating in black culture (Anderson et al. 1996, El-Kebbi et al. 1996). The need for strong family support when following a diet and dissatisfaction with health providers has also been mentioned in other focus groups with black adults (Maillet et al. 1996). Other studies suggest that health providers may not be providing enough diabetes-related information to their patients (Jefferson et al. 2000). 

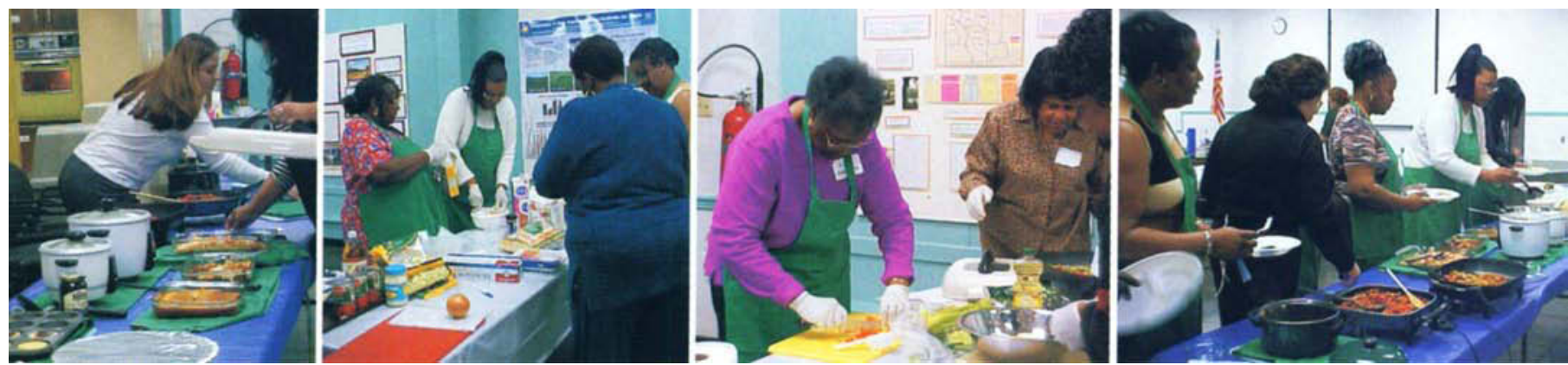

Information gathered in the focus groups is being incorporated into a pilot outreach program - a series of cooking workshops targeting African Americans. About two-dozen community members attended a recipe-testing workshop sponsored by UC Cooperative Extension in San Joaquin County, where they prepared, sampled and gave researchers feedback on healthier versions of traditional African American recipes.

The faith community or church is increasingly recognized as an important channel for health promotion messages targeting African Americans (Oexmann et al. 2000, McNabb et al. 1997). Black women with diabetes are almost four times as likely as white women to receive diabetes information through their churches (Schoenberg 1998). A community-based program, delivered by trained lay volunteers through the churches, can be as effective as clinic-based programs in achieving weight loss in African Americans (McNabb et al. 1997).

Based on these findings, the Health Promotion workgroup is now planning a multichannel campaign, working closely with the African American community. The components include: cooking classes using modified African American recipes; opportunities in small group settings to talk with health providers about diabetesrelated services; and visual messages disseminated through the African American community via clinics, schools and churches. The goals of this campaign and related activities are to raise the level of diabetes awareness and promote earlier screening and treatment for the disease. A secondary goal will be to motivate people to adopt healthier lifestyles that reduce the risk of developing type 2 diabetes.

UC Health Promotion Workgroup members who authored in this study are: Lucia L. Kaiser, Marilyn S. Townsend, Francene M. Steinberg and Amy B. Joy, Department of Nutrition, UC Davis;
Estella A. West, UC Cooperative Extension (UCCE) Santa Clara County; Gloria R. Brown, UCCE San Mateo County; Nancy I. Feldman, UCCE Stanislaus County; Connie L. Garrett, UCCE San Bernardino County; Anna Martin and Anna Olivares, UCCE San Joaquin County; Edwina U. Williams, UCCE Los Angeles County; Christine M. Bruhn, Department of Food Science and Technology, UC Davis; and Marciel A. Klenk, UCCE Napa County.

\section{References}

Airhihenbuwa $\mathrm{CO}$, Kumanika S, Agurs D, et al. 1996. Cultural aspects of African American eating patterns. Ethnicity Health 1(3):245-60.

Anderson RM, Barr PA, Edwards GJ, et al. 1996. Using focus groups to identify psychosocial issues of urban black individuals with diabetes. Diabetes Educator 22(1):28-33.

Brancati FL, Kao LWH, Folsom AR, et al. 2000 . Incident type 2 diabetes mellitus in African American and white adults. JAMA 283(17):2253-9.

Brown JB, Nichols GA, Glauber HS, Bakst AW. 1999. Type 2 diabetes: Incremental medical care costs during the first 8 years after diagnosis. Diabetes Care 22(7):116-24.

Diabetes Prevention Program Research Group. 2002. Reduction in the incidence of type 2 diabetes with lifestyle intervention or metformin. New England J Med 346(6):393-403.

Diabetes Research Working Group. 1999. Summary of the report and recommendations of the Congressionally established Diabetes Research Working Group. www.diabetes.org/ada/drwg/

drwgsummary.html.

EI-Kebbi M, Bacha GA, Ziemer DC, et al. 1996. Diabetes in urban African Americans V: Use of discussion groups to identify barriers to dietary therapy among low-income individuals with non-insulin-dependent diabetes mellitus. Diabetes Educator 22(5):488-92.

Gaillard TR, Schuster DP, Bossetti BM, et al. 1997. Do sociodemographics and eco- nomic status predict risks for type II diabetes in African Americans? Diabetes Educator 23(3):294-300.

Harris MI, Flegal KM, Cowe CC, et al. 1998. Prevalence of diabetes, impaired fasting glucose, and impaired glucose tol erance in U.S. adults. Diabetes Care 21(4):518-24.

Hosey G, Gordon S, Levine A. 1998. Type 2 diabetes in people of color. Nurse Practitioner Forum 9(2):108-14.

Jefferson VW, Melkus GD, Spollett GR. 2000 . Health-promotion practices of young black women at risk for diabetes. Diabetes Educator 26(2):295-302.

Krieger N, Sidney S. 1996. Racial discrimination and blood pressure: The CARDIA study of young black and white adults. Am J Public Health 86(10):1370-8.

Krueger RA. 1994. Focus Groups. Thousand Oaks, CA: Sage Publ. $255 \mathrm{p}$.

Maillet NA, Melkus GD, Spollett GR. 1996. Using focus groups to characterize the health beliefs and practices of black women with non-insulin-dependent diabetes. Diabetes Educator 22(1):39-46.

McNabb W, Quinn M, Tobian J. 1997. The Pathways church-based weight loss program for urban African American women at risk for diabetes. Diabetes Care 20(10): 1518-23.

Mokdad AH, Ford ES, Bowman BA, et al. 2000. Diabetes trends in the U.S.: 19901998. Diabetes Care 23(9):1278-83.

Oexmann MJ, Thomas JC, Taylor KB, et al. 2000. Short-term impact of a churchbased approach to lifestyle change on cardiovascular risk in African Americans. Ethnicity Dis 10:17-23.

Osei K, Cottrel DA, Ezenwaka EC, et al. 1993. Serum insulin and glucose concentrations in people at risk for type II diabetes. A comparative study of African Americans and Nigerians. Diabetes Care 16(10):1367-75.

Pi-Sunyer FX. 1990. Obesity and diabetes in blacks. Diabetes Care 13(11, suppl 4):1144-9.

Schneider D, Greenberg M, Lu L. 1997. Early life experiences linked to diabetes mellitus: A study of African American migration. J Nat Med Assoc 89:29-34.

Schoenberg $\mathrm{NE}$, Amey $\mathrm{CH}$, Coward RT. 1998. Diabetes knowledge and sources of information among African American and white older women. Diabetes Educator 24(3):319-24. 